\title{
VARIATIONAL METHODS FOR THE SOLUTION OF PROBLEMS OF EQUILIBRIUM AND VIBRATIONS
}

\author{
R. COURANT
}

As Henri Poincaré once remarked, "solution of a mathematical problem" is a phrase of indefinite meaning. Pure mathematicians sometimes are satisfied with showing that the non-existence of a solution implies a logical contradiction, while engineers might consider a numerical result as the only reasonable goal. Such one sided views seem to reflect human limitations rather than objective values. In itself mathematics is an indivisible organism uniting theoretical contemplation and active application.

This address will deal with a topic in which such a synthesis of theoretical and applied mathematics has become particularly convincing. Since Gauss and W. Thompson, the equivalence between boundary value problems of partial differential equations on the one hand and problems of the calculus of variations on the other hand has been a central point in analysis. At first, the theoretical interest in existence proofs dominated and only much later were practical applications envisaged by two physicists, Lord Rayleigh and Walther Ritz; they independently conceived the idea of utilizing this equivalence for numerical calculation of the solutions, by substituting for the variational problems simpler approximating extremum problems in which but a finite number of parameters need be determined. Rayleigh, in his classical work-Theory of sound - and in other publications, was the first to use such a procedure. But only the spectacular success of Walther Ritz and its tragic circumstances caught the general interest. In two publications of 1908 and 1909 [39], Ritz, conscious of his imminent death from consumption, gave a masterly account of the theory, and at the same time applied his method to the calculation of the nodal lines of vibrating plates, a problem of classical physics that previously had not been satisfactorily treated.

Thus methods emerged which could not fail to attract engineers and physicists; after all, the minimum principles of mechanics are more suggestive than the differential equations. Great successes in applications were soon followed by further progress in the understanding of the theoretical background, and such progress in turn must result in advantages for the applications.

An address delivered before the meeting of the Society in Washington, D.C., on May 3, 1941, by invitation of the Program Committee; received by the editors June $16,1942$. 
The following address will try to convey an idea of this interplay between practical and theoretical points of view. Completeness cannot be attempted; rather some selected topics with which the speaker has been personally concerned will be discussed.

Usually the solution of a difficult problem in analysis proceeds according to a general scheme: The given problem $P$ with the solution $S$ is replaced by a related problem $P_{n}$ so simple that its solution $S_{n}$ can be found with comparative ease. Then by improving the approximation $P_{n}$ to $P$ we may expect, or we may assume, or we may prove, that $S_{n}$ tends to the desired solution $S$ of $P$. The essential point in an individual case is to choose the sequence $P_{n}$ in a suitable manner.

Here we shall deal mainly with problems of equilibrium and vibrations (boundary value and eigenvalue problems, ${ }^{1}$ respectively). They lead to linear self-adjoint differential equations for an unknown function $u(x, y)$ :

$$
L(u)=f,
$$

or

$$
L(u)+\lambda u=0,
$$

in a two-dimensional domain of the $x, y$-plane, or rather to equivalent variational problems for the kinetic and potential energies of the system.

\section{The VARIATIONAL PROBLEMS}

We assume our domain $B$ bounded by a piecewise smooth curve $C$, and we denote the arc length measured along $C$ by $s$, and differentiation in the direction of the inward normal by $\partial / \partial n$ or by a subscript $n$.

1. Quadratic functionals. Our variational problems refer to quadratic functionals

$$
Q(v)=Q(v, v)
$$

defined by symmetric bilinear expressions such as

$$
\begin{aligned}
\text { (3) } D(v, w) & =\iint_{B}\left(v_{x} w_{x}+v_{y} w_{y}\right) d x d y, \\
\text { (4) } M(v, w) & =\iint_{B}\left[\Delta v \Delta w+\alpha\left(v_{x x} w_{y y}+v_{y y} w_{x x}-2 v_{x y} w_{x y}\right)\right] d x d y,
\end{aligned}
$$

${ }^{1}$ Problems of critical loads (buckling) are likewise mathematically formulated as eigenvalue problems. 
which occur in the study of membranes and plates, respectively. In (4) the symbol $\Delta$ denotes the Laplacian operator, and $\alpha$ a constant. Furthermore, we shall have to use other quadratic integrals defined by bilinear expressions such as

$$
\begin{aligned}
& H(v, w)=\iint_{B} v w d x d y, \\
& K(v, w)=\int_{C} v w d s, \\
& R(v, w)=\int_{L} v_{x x} w w_{x x} d x,
\end{aligned}
$$

where $L$ is a line $y=$ const. in $B$ and $C$ is the boundary of $B$. We then consider functionals such as

$$
Q(v, w)=a D(v, w)+b M(v, w)+c K(v, w)+d R(v, w),
$$

where $a, b, c, d$ are constants. Always the "admissible" functions $u, v$, $w, \cdots$ are restricted by the condition that all the occurring integrands be at least piecewise continuous.

The stable equilibrium of a plate or membrane under an external pressure $f$ is characterized by a variational problem of the type

$$
Q(v)+2 H(v, f)=\text { minimum, }
$$

for the deflection $v$, whereas vibrations of plates and membranes correspond to the problem of finding stationary values, $\nu^{2}=\lambda$, of

$$
Q(v) / H(v) \text {. }
$$

The values $\nu$ thus defined are the natural frequencies of the system. $Q(v)$ corresponds to the potential energy of the system in the case of equilibrium, while for vibrations $Q(v)$ and $H(v)$ are the "reduced" potential and kinetic energies, respectively. ${ }^{2}$ Terms of the form $K$ and $R$ appearing in the expression $Q$ represent additional energies concentrated along the boundary $C$ of $B$ or along a line $L$ in $B$. For example, in the case of reinforced plates we would have $Q=M+d R$ if the reinforcement consists of a bar along the line $L$.

2 By "reduced" energies we mean the following: If we assume the free system vibrating with a frequency $\omega$ (not necessarily a natural frequency), then the deflection $u$ may be represented in the form $u=v \cos \omega t$, where $v$ is a function of position only. The potential energy $V$ may then be written in the form $Q(v) \cos ^{2} \omega t$ and the kinetic energy $T$ in the form $\omega^{2} H(v) \sin ^{2} \omega t$; the quantities $Q$ and $H$ are termed the reduced energies. 
2. Rigid and natural boundary conditions. (See $[1,2,9]$.) The Euler differential equations (1) or (2) of our variational problems must be supplemented by appropriate boundary conditions. If we focus our attention on the differential equations as such, it is not at all obvious what boundary conditions belong to a particular problem of mechanics. However, from the point of view of the calculus of varfations a complete clarification of this delicate question is almost automatically obtained if the mathematical reasoning follows our mechanical intuition. In the first place we observe that in a variational problem (not so in a problem of differential equations) we need not in advance impose boundary conditions in order to single out a specific solution. If the functions admissible in the competition are not subjected to restrictions at the boundary, we speak of a "free problem." In these problems the first variation of the functional will contain terms referring to the boundary, and the vanishing of the first variation will imply not only Euler's differential equation for the domain $B$ but also conditions on the boundary $C$, which we call "natural boundary conditions." Now the dominant fact is: appropriate boundary conditions for differential equations are obtained as natural boundary conditions of corresponding variational problems. In the latter they may, but they need not be prescribed in advance.

There is only the exceptional case, often termed the "simplest case of a variational problem" of fixed boundary values of $u$ or derivatives of $u$ or other expressions in $u$. Here the situation seems somewhat obscured. (The clamped membrane with the boundary condition $u=0$ and the clamped plate with the boundary conditions $u=u_{x}=u_{y}=0$ belong in this category.) Such fixed or rigid or artificial boundary conditions must be explicitly stipulated for the variational problem not only for the differential equation. However, we shall recognize them as limiting cases or degenerations of natural conditions.

Physically, rigid conditions correspond to rigid constraints of the system at the boundary $C$ while natural conditions express equilibrium of the system of $C$ if along $C$ partial or full freedom of motion is permitted.

To understand the significance of natural boundary conditions the following observation is essential: The Euler differential equations depend only on the domain integrals or the energies spread over $B$. But the natural boundary conditions are essentially affected by the boundary integrals representing those contributions to the energies which are concentrated along the boundary $C$. These terms lead to a great variety of possible natural boundary conditions for the same differential equation. In a somewhat different way we may formulate 
the fact: Natural boundary conditions, but not Euler's equations, are affected by divergence expressions in the domain integrals.

2a. Examples. Plates, plane torsion for multiply-connected domains. As an example we consider a membrane with a free boundary. A pressure $f$ of average value zero may act on the membrane. In this case we have a variational problem for $D(v)+2 H(v, f)$; if $\zeta$ denotes an arbitrary variation of $v$, the variational condition is

$$
D(v, \zeta)+H(\zeta, f)=0 .
$$

Transforming $D(v, \zeta)$ by Green's formula into a domain integral plus a boundary integral, we find the natural boundary condition

$$
\frac{\partial v}{\partial n}=0,
$$

expressing the fact that no force acts on the boundary $C$. For a plate, free at the boundary, $Q(v)=M(v)$, and the natural boundary conditions appear as the classical Kirchhoff conditions:

$$
\begin{aligned}
(1+\alpha) \Delta v & =\alpha\left(v_{x x} x_{n}^{2}+2 v_{x y} x_{n} y_{n}+v_{y y} y_{n}^{2}\right), \\
\frac{\partial}{\partial n} \Delta v & =\alpha\left(v_{x x} x_{n} x_{s}+v_{x y}\left[x_{n} y_{s}+x_{s} y_{n}\right]+v_{y y} y_{n} y_{s}\right),
\end{aligned}
$$

where $x_{n}, y_{n}$ and $x_{s}, y_{s}$ are, respectively, direction cosines of the inner normal and tangent vector along $C$.

In $M(v)$ the term $\alpha\left(v_{x x} v_{y y}-v_{x y}^{2}\right)=\alpha \partial\left(v_{x} v_{y y}\right) / \partial x-\alpha \partial\left(v_{x} v_{x y}\right) / \partial y$ is a divergence expression, very essential for Kirchhoff's natural boundary conditions but irrelevant for Euler's differential equation $\Delta \Delta v=0$ and without consequence for the clamped plate.

Similar remarks apply to natural "discontinuity conditions" that arise if energy is concentrated along lines $L$ interior to $B$, such as in the case of reinforced plates. For example, for a rectangular plate clamped at the boundary but reinforced by a bar along the line $y=0$, the variational problem becomes

$$
\begin{aligned}
Q(v)+2 H(v, f)+k R(v)= & \iint_{B}\left[(\Delta v)^{2}+2 \alpha\left(v_{x x} v_{y y}-v_{x y}^{2}\right)\right] d x d y \\
& +k \int_{L} v_{x x}^{2} d x+2 \iint_{B} v f d x d y=\min .
\end{aligned}
$$

with the condition that $v=v_{x}=v_{y}=0$ on $C$ and $v, v_{x}, v_{y}$ are continuous in $B$ while the second derivatives of $v$ are at least piecewise continu- 
ous in $B$. If $\zeta$ is an arbitrary variation of $v$ satisfying these conditions, we obtain as above not only the Euler equation $\Delta \Delta v+f=0$, but also the natural discontinuity condition

$$
\left[\left(\Delta v+\alpha v_{x x}\right)\right]_{-}^{+}=\frac{\partial^{4}}{\partial x^{4}} v(x, 0),
$$

where the symbol $[g]_{-}^{+}$means the amount of discontinuity suffered by a function $g$ in crossing the line $y=0$ from positive to negative values of $y$. Another example of a somewhat different character is the the problem of torsion of long columns with multiply connected cross sections. The contour $C$ of the cross section may include a domain $B$ in the $x, y$-plane from which are removed holes $B_{1}, B_{2}, B_{3}, \cdots$ with contours $C_{1}, C_{2}, C_{3}, \cdots$ and areas $A_{1}, A_{2}, A_{3}, \cdots$. The multiplyconnected domain between $C$ and $C_{1}, C_{2}, C_{3}, \cdots$ may be called $B^{*}$.

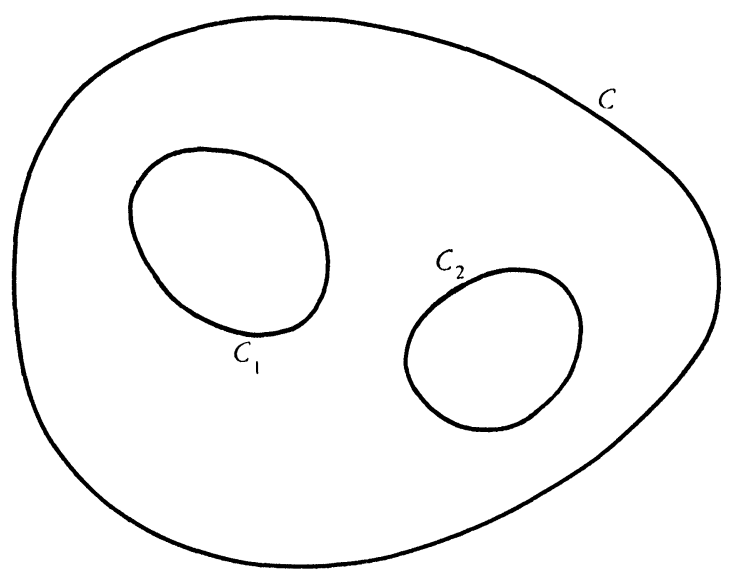

FIG. 1

Then the adequate variational formulation of the torsion problem in proper units is: To find a function $\phi=u$ continuous in $B+C$, having piecewise continuous first derivatives in $B$, having the boundary values zero on $C$ and constant, but not prescribed values $c_{i}$ in the holes $B_{i}$, such that for the whole domain $B$

$$
D(\phi)=\iint\left[\left(\phi_{x}^{2}+\phi_{y}^{2}\right)+2 \phi\right] d x d y
$$

attains its least value $d$ for $\phi=u$. The function $u$ then will give the stresses in the cross section by differentiation. 
Note that this problem requires not only to determine $u$ in $B^{*}$ as a solution of Euler's equation

$$
\Delta u=1
$$

with the boundary conditions $u=0$ on $C$ and $u=$ const. $=c_{i}$ on $C_{i}$, but also to find the constants $c_{i}$. The freedom for these parameters in the functions $\phi$ admissible in the variational problem must lead to natural conditions from which the $c_{i}$ are to be characterized.

Indeed, from the variational condition $D(u, \zeta) \times \iint \zeta d x d y=0$ valid for arbitrary $\zeta$ that vanishes on $C$ and has constant values in each $B$ : we can only obtain Euler's equation, but likewise immediately the natural boundary conditions

$$
\int_{C_{i}} \frac{\partial u}{\partial n} d s+c_{i} A_{i}=0
$$

which connect the unknown boundary constants with the known areas $A_{i}$.

Incidentally, for the special choice $\zeta=u$ we obtain (in line with more general results) that the solution, representing a state of equilibrium, satisfies the relation

$$
S=D(u)=-\iint u d x d y .
$$

The quantity $S$ represents the "total stiffness" of the column with respect to torsion.

In the appendix we shall see how this problem, which as an ordinary boundary value problem of a partial differential equation would be rather formidable, can be attacked numerically with success from the point of view developed here.

2b. Rigid constraints as limiting cases. If we have additional energy concentrated at the boundary and expressed, for example, by the term $K(v)=\int_{C} v^{2} d s$, then not the Euler equation but the natural boundary conditions will be influenced by these terms. Thus, for the free membrane with

$$
Q(v)=D(v)+\gamma K(v)
$$

we obtain the natural boundary condition

$$
\frac{\partial v}{\partial n}-\gamma v=0
$$

We observe that as the parameter $\gamma$ increases indefinitely, that is, as 
the elastic restoring forces at the boundary approach a rigid constraint, the condition (10) tends to the boundary condition $v=0$ of the clamped membrane. This is in harmony with the fact that for large positive $\gamma$ the boundary values of $v^{2}$ though free, must in the average be small in order to keep the energy $Q(v)$ in bounds.

Quite generally rigid boundary conditions should be regarded as limiting cases of natural conditions in which a parameter tends to infinity. This corresponds to the physical fact that rigid constraints are only idealized limiting cases of very large restoring forces. ${ }^{3}$ It may be mentioned that this interpretation of fixed boundary conditions is the key to a more penetrating analysis of the question what we may and what we may not prescribe at the boundary (see [1]).

3. General conclusions. The mere formulation of our problem in terms of maxima and minima leads to further important applications. As an example we mention a famous principle first formulated by Lord Rayleigh [38]: If a vibrating system whose energies are expressed by quadratic integrals is changed into another system by an increase of masses or by a decrease of elastic forces, then all the natural frequencies of the syste can only change toward lower values. This fact was recognized by the speaker (see [9]) as a consequence of the following theorem, which is easily proved: The $n$th natural frequency of any of our vibration problems is the highest value of the lowest frequency of all systems obtained from the given system by imposing $n-1$ constraints; or the $n$th eigenvalue $\lambda_{n}$ is the largest value attained by the minimum $d\left(w_{1}, \cdots, w_{n-1}\right)$ of the quotient $Q(v) / H(v)$ if $v$ is subjected to $n-1$ linear conditions of the form

$$
H\left(v, w_{i}\right)=0, \quad i=1, \cdots, n-1,
$$

the $w_{i}$ being arbitrarily chosen functions. Here "minimum" refers to a fixed set of $n-1$ functions $w_{i}$.

This principle can render useful service for appraising the change in the natural frequencies resulting from changes in the given vibrating system. We recognize immediately that a stiffening of the system by the introduction of new elastic forces (which lead to an increase in potential energy) must produce higher natural frequencies throughout. Likewise, imposing new rigid constraints will have the same effect. Even quantitative results can be obtained by a simple applica-

\footnotetext{
${ }^{3}$ It might be mentioned that A. Weinstein's method (see $\$ 3$ ) is a somewhat different way of presenting rigid boundaries as limiting cases of problems with fewer restrictions.
} 
tion of this principle. The asymptotic behavior of the natural frequencies $\nu_{n}=\lambda_{n}^{1 / 2}$ for large $n$ was found by A. Sommerfeld, H. Weyl, and the speaker. For example, the $n$th eigenvalue of $\Delta v+\lambda v=0$ for a free or clamped boundary and domain of area $A$ behaves asymptotically like $4 \pi n / A$. (See [9].)

Recently Alexander Weinstein has made an interesting application of this maximum-minimum principle to clamped plates [4]. The eigenvalue problem for a "supported" plate (with $\alpha=0$ ) refers to the quadratic energy expressions $Q(v)=\iint_{B}(\Delta v)^{2} d x d y, H(v)=\iint_{B} v^{2} d x d y$. It is then required to make the quotient $Q / H$ stationary only under the boundary condition $v=0$. From this variational problem, we obtain the Euler equation $\Delta \Delta v-\lambda v=0$ and one natural boundary condition, $\Delta v=0$; the solutions for this problem are in this case identical with those of the clamped membrane. Now Weinstein, by imposing successively a denumerable number of boundary conditions of the form $\int_{C}(\partial v / \partial n) \phi_{i} d s=0, i=1,2,3, \cdots$, where the $\phi_{i}$ form a complete system of functions on the boundary $C$, obtains the problem of the clamped plate as a limiting case. The approximating problems stipulate conditions less restrictive than the limiting problem of a clamped plate, consequently they lead to smaller values of the minima and hence of the maxi-minima, and therefore provide lower bounds for the natural frequencies of the clamped plate. It is remarkable that the approximating problems could be solved explicitly in terms of solutions of the membrane problem $[20,21,22]$.

\section{Rayleigh-Ritz method}

We now discuss the question of attacking a variational problem numerically. In principle, there are many ways in which such a variational problem may suggest approximations by simpler problems. The Rayleigh-Ritz method is only one of them.

1. The principle and theoretical aspects. Suppose we seek the minimum $d$ of an integral expression or any other variational expression $I(\phi)$ (for example, our quadratic functionals of the preceding section). We then start with a minimizing sequence

$$
\phi_{1}, \phi_{2}, \phi_{3}, \cdots, \phi_{n}, \cdots \text {, }
$$

that is, a sequence of functions, admissible in our variational problem, for which

$$
\lim _{n \rightarrow \infty} I\left(\phi_{n}\right)=d
$$


$d$ being the lower bound of the functional $I(\phi)$. The existence of the lower bound $d$ is obvious or may be easily proved in all relevant problems and the existence of the minimizing sequence (11) is then a logical consequence.

However, the problem in applications is one, not of the existence, but of the practical construction of such a minimizing sequence. Ritz's method is nothing but a recipe for such a construction. A minimizing sequence immediately furnishes an approximation to $d$ (sometimes this is all we wish to know, for example, if we are interested in the natural frequencies of a vibrating system). Moreover, it may be assumed and in many cases it can be proved that the minimizing sequence itself will furnish a good approximation for the function $u$ which actually solves the problem.

Ritz's construction proceeds as follows: We start with an arbitrarily chosen system of "coordinate functions"

$$
\omega_{1}, \omega_{2}, \cdots, \omega_{n}, \cdots \text {, }
$$

which should satisfy the two conditions:

(a) Any linear combination

$$
\phi_{n}=c_{1} \omega_{1}+c_{2} \omega_{2}+\cdots+c_{n} \omega_{n}
$$

of them is admissible in the variational problem.

(b) They should form a complete system of functions in the sense that any admissible function $\phi$ and its relevant derivatives may be approximated with any degree of accuracy by a linear combination of coordinate functions and of their corresponding derivatives, respectively.

If we begin with such a system of coordinate functions, it is clear that for $n$ sufficiently large and for a suitable choice of the coefficients $c_{1}, c_{2}, \cdots, c_{n}$ of (14) we can find admissible functions $\phi_{n}$ for which $I\left(\phi_{n}\right)$ differs arbitrarily little from $d$. In other words, it is possible to find a minimizing sequence $\phi_{1}, \phi_{2}, \cdots, \phi_{n}, \cdots$ as a sequence of linear combinations of the coordinate functions. In order to obtain such a minimizing sequence we choose the $c_{i}$ in the following manner. We consider any function $\phi_{n}$ defined by (14) and substitute it in our variational problem. $I\left(\phi_{n}\right)$ then becomes a function $F\left(c_{1}, \cdots, c_{n}\right)$ of the $n$ parameters $c_{i}$ which we may now determine from the ordinary minimum problem of the calculus

$$
I\left(\phi_{n}\right)=F\left(c_{1}, \cdots, c_{n}\right)=\min .
$$

In the problems considered here, $I(\phi)$ is a quadratic or bilinear functional, and (15) leads to a system of $n$ linear equations for the 
parameters, ${ }^{4}$ a system which may be solved by established methods. Thus the minimizing sequence, $\phi_{n}(n=1,2, \cdots)$ is found. Recalling our general scheme we may identify the construction of the $\phi_{n}$ with the problem $P_{n}$.

At this point one important remark may be made [1]. While the convergence of $I\left(\phi_{n}\right)$ to $d$ is assured, it is by no means generally true that $\phi_{n}$ tends to $u$, the solution of the original minimum problem, or, even less so, that the derivatives of $\phi_{n}$ tend to the corresponding derivatives of $u$. However, a comparative investigation of different types of variational problems reveals that, generally speaking, the convergence of a minimizing sequence $\phi_{1}, \phi_{2}, \cdots, \phi_{n}, \cdots$ and the sequences of the derivatives of the $\phi_{n}$ is improved if the order of the occurring derivatives becomes higher. On the other hand, there is a tendency toward worse convergence as the number of independent variables increases. For example, in the one-dimensional problem of an elastic string $\left(Q(v)=\int_{0}^{s} v^{\prime 2} d x\right)$, the convergence of the $\phi_{n}$ to $u$ is assured, the derivatives $\phi_{n}^{\prime}$, however, need not converge to $u^{\prime}$. But for the corresponding problem of the bar $\left(Q(v)=\int_{0}^{s} v^{\prime \prime 2} d x\right)$, not only does $\phi_{n}$ converge to $u$ but also $\phi_{n}^{\prime}$ to $u^{\prime}$. On the other hand, in the membrane problem even the $\phi_{n}$ need not converge to $u$, while in the case of the plate the convergence of $\phi_{n}$ to $u$ is assured. The first success attained by Ritz depended largely on his good fortune in attacking the seemingly more difficult problem of the plate rather than that of the membrane.

These facts which are intimately related to more profound questions in the general theory of the variational calculus have suggested the following method of obtaining better convergence in the Rayleigh-Ritz method. Instead of considering the simple variational problem for the corresponding boundary value problem, we modify the former problem without changing the solution of the latter. This is accomplished by adding to the original variational expression terms of higher order which vanish for the actual solution $u$. For

4 This suggests the following interpretation and generalization of the procedure whereby reference to a minimum problem need no longer be made: we replace our differential equation $L(u)=0$ by the condition that $L(u)$ should be orthogonal to $n$ functions of a previously selected complete system of functions. If, then, $n$ tends to infinity, the totality of all these relations will be substituted for the differential equation, and for any fixed $n, u$ may be chosen, for example, as a linear combination (14): $u=\phi_{n}$. In this general pattern that goes back to Galerkin, there is more freedom left for the choice of the approximations to the solution $u$. However, in the Rayleigh-Ritz method proper, generally speaking the question of convergence is more easily investigated. (The generalized view interpretation is implicitly mentioned in Ritz' papers and was later developed by several authors. See for example $[18,19]$.) 
example, we may formulate the equilibrium problem for a membrane under the external pressure $f$ as follows:

$$
I(v)=\iint_{B}\left(v_{x}^{2}+v_{y}^{2}+v f\right) d x d y+\iint_{B} k(\Delta v-f)^{2} d x d y=\min .,
$$

where $k$ is an arbitrary positive constant or function. Such additional terms make $I(v)$ more sensitive to variations of $v$ without changing the solution. In other words, minimizing sequences attached to such a "sensitized" functional will by force behave better as regards convergence [7].

The practical value of the method of sensitizing the integral by the addition of terms of higher order has not yet been sufficiently explored. Certainly the sensitizing terms will lead to a more complicated system of equations for the $c_{i}$. This means that a compromise must be made for a suitable choice of the arbitrary positive function $k$ so that good convergence is assured while the necessary labor is kept within bounds.

2. Practical viewpoints. Theoretically the Rayleigh-Ritz method consists merely in the construction of the minimizing sequence. However, the difficulty that challenges the inventive skill of the applied mathematician is to find suitable coordinate functions and to estimate the accuracy of the result. From a practical point of view almost any success depends on the selection of coordinate functions. If these functions are chosen without proper regard for the individuality of the problem the task of computation will become hopeless. A choice should be made so that the system of linear equations for the $c_{i}$ obtained from (15) will have a preponderance of terms along the diagonal of their matrix, and that the number of terms to be taken into account be kept small. Since only a few of the coordinate functions will enter into the calculation, the theoretical completeness of the $\phi_{n}$ is irrelevant. It is important that the initial function be already a fair approximation, and furthermore that the functions $\phi_{1}$, $\phi_{2}, \cdots$ should be sufficiently differen $t^{5}$ so that increasing the number of terms will lead to an actual improvement of the approximation. Importance should also be attached to the need for making the numerical evaluation of the coefficients of our linear equations practicable.

In many cases the use of polynomials for coordinate functions is most advantageous. Si Luan Wei has shown in his thesis that results

${ }^{5}$ Such a "difference" can be measured. See Courant-Hilbert, Methoden der mathemateschen Physik, vol. I, p. 52. 
for plates can be obtained by polynomials more readily and more accurately than by the coordinate functions originally used by Ritz [40].

Recently physicists have chosen coordinate functions according to the following pattern. One starts with a choice of the function $\omega_{1}$, which is expected to be a fair approximation to the actual solution. Then $\omega_{2}$ is defined by $\omega_{2}=L\left(\omega_{1}\right)$. Finally one chooses $\omega_{3}=L\left(\omega_{2}\right)$ $=L L\left(\omega_{1}\right), \omega_{4}=L\left(\omega_{3}\right)=L L L\left(\omega_{1}\right)$, and so on. This choice makes the calculation of the matrix elements of our linear equations for the $c_{i}$ comparatively simple. Since the operator $L$ introduces higher derivatives which may lead to cumbersome complications at boundaries, it is understandable that such a choice of the $\omega_{n}$ seems feasible primarily for infinite domains where no boundary is given and where the natural boundary conditions are equivalent to the finiteness of the variational integrals.

3. Boundary conditions. For rigid boundary conditions the approximation by the Rayleigh-Ritz method is comparatively good. Few admissible coordinate functions would in most cases suffice to yield a result near the desired solution. This pleasant feature, however, is offset by the restriction imposed by the rigid boundary conditions which in general precludes the choice of simple coordinate functions.

For free boundaries and natural boundary conditions the choice of coordinate functions is eased considerably since no boundary conditions need be stipulated in advance. As a general rule we might well use, for the $\phi_{n}$, polynomials with undetermined coefficients. For this great advantage, however, we must pay a price, namely, the necessity for using many more terms to secure reasonable accuracy. Therefore it is sometimes preferable if we can satisfy in advance, at least approximately, the natural boundary conditions, by a proper choice of functions. Still, the advantage gained by making the $\phi_{n}$ polynomials might be decisive.

In this connection it seems to be of importance that rigid boundaries can be considered as a limiting case of free boundaries, as indicated previously in $\$ I, 2$. As an example we may consider the following problem for a membrane:

$$
\begin{aligned}
D(v)+2 H(v, f)+\gamma K(v)= & \iint_{B}\left(v_{x}^{2}+v_{y}^{2}+2 v f\right) d x d y \\
& +\gamma \int_{C} v^{2} d s=\min .
\end{aligned}
$$


If $\gamma$ is very large, then the free boundary problem relating to this expression is almost identical to the corresponding problem for a clamped membrane. By letting $\gamma$ tend to infinity we "freeze" the boundary. This suggests an attempt to solve the equilibrium problem of the clamped plate by choosing a large numerical value for $\gamma$ and then treating the problem as one for a free membrane. However, to obtain reasonable accuracy for the free problem, the larger the quantity $\gamma$ the more terms will be necessary. Hence the practical application of the method again requires a compromise $-\gamma$ must be chosen large enough to approximate rigidity but small enough to keep the necessary labor within reasonable bounds. From a theoretical as well as from a practical point of view it would seem worth while to study the preferable choices of these artificial parameters.

4. Estimates. A weak point in the Rayleigh-Ritz procedure is that it does not contain a principle for estimating the accuracy of the approximation. This is not the place to give an account of the numerous efforts made to fill this gap at least theoretically. For problems of equilibrium, estimates for the minimum $d$ can be obtained by a method suggested by Castigliano's principle in the theory of elasticity. The mathematical idea is to represent the minimum value $d$ of the given problem as a maximum value of another variational problem (see [28]). The margins obtained are quite narrow. But the practical value of this accuracy does not appear great since the value of $d$ in problems of equilibrium is of little interest, ${ }^{6}$ whereas we are concerned with the deflection $u$ and its derivatives. In the case of vibrations, where the minimum values, as squares of the frequencies, are of considerable importance, methods for the estimation of accuracy are in general less satisfactory [29].

5. Objections to the Rayleigh-Ritz method. The vagueness as to the accuracy of the approximation obtained is only one of the objections to the Rayleigh-Ritz method that may be raised. More annoying is that a suitable selection of the coordinate functions is often very difficult and that laborious computations are sometimes necessary. For these reasons, alternative methods must be studied.

\section{Method of Finite differences. General RANDom STATISTICAL METHODS}

As far as practical experience goes the most important of these

\footnotetext{
${ }^{6}$ See, however, E. Trefftz, Math. Ann. vol. 108 (1933), p. 595 where the deflection at a point $x_{0}, y_{0}$ is represented as such a minimum $d$ of a modified functional.
} 
methods is that of finite differences. In this well known procedure we replace differential quotients by difference quotients and integral expressions by finite sums defined over a set of net-points in the plane, for example, a quadratic net in the domain $B$ formed by the intersection of the lines $x=\gamma h, y=\mu h(\gamma=1,2, \cdots, N ; \mu=1,2, \cdots, M)$. The simplified problem $P_{n}$ of difference equations can very often be solved with relative ease. Then if we permit the mesh, $h=1 / n$, of the net to tend to zero with increasing $n$, not only does $P_{n}$ tend to $P$, but the solutions $S_{n}$ of the difference equations approach the solution $S$ of the original problem exceedingly well. Furthermore, we have the remarkable fact that all relevant difference quotients of first and higher order converge to the corresponding derivatives pertaining to the original problem.

On these grounds the method of finite difference as a general procedure is often preferable to the Rayleigh-Ritz method. The latter might lend itself more readily to the solution of specific problems where suitable analytic expressions are available for coordinate functions. However, in other cases experience points to the superiority of the method of finite differences. One of the underlying reasons is that finite differences are attached directly to the values of the function itself without an interceding medium such as the more or less arbitrary coordinate functions.

If the variational problems contain derivatives not higher than the first order the method of finite difference can be subordinated to the Rayleigh-Ritz method by considering in the competition only functions $\phi$ which are linear in the meshes of a sub-division of our net into triangles formed by diagonals of the squares of the net. For such polyhedral functions the integrals become sums expressed by the finite number of values of $\phi$ in the net-points and the minimum conditions become our difference equations. Such an interpretation suggests a wide generalization which provides great flexibility and seems to have considerable practical value. Instead of starting with a quadratic or rectangular net we may consider from the outset any polyhedral surfaces with edges over an arbitrarily chosen (preferably triangular) net. Our integrals again become finite sums, and the minimum condition will be equations for the values of $\phi$ in the net-points. While these equations seem less simple than the original difference equations, we gain the enormous advantage of better adaptability to the data of the problem and thus we can often obtain good results with very little numerical calculation. (See appendix.)

This procedure of finite differences was analyzed from a mathematical point of view-and in particular the convergence for the quad- 
ratic mesh width $h \rightarrow 0$ was proved-first by Philipps in unpublished papers, then by Philipps and Wiener [14], and at the same time by Lusternik and by Courant [5], and later by Courant, Friedrichs, and Lewy [8].

As was already observed by Philipps and Wiener, equations of finite differences can be interpreted by processes of probability-the so-called random walk. This connection has led Courant, R. Lueneburg [16], and Petrovsky [17] to a more general attack upon our problems, as follows: Instead of simply replacing our integral expressions by finite sums defined over a net, we may replace them in various ways by other simple functionals and thus obtain a greater variety of possible approximate problems $P_{n}$. Consider, for example, the nonnegative function $K(x, y ; \xi, \eta)$ which is assumed to be symmetric, to be defined and piecewise continuous in the entire plane and to satisfy $\iint_{-\infty}^{+\infty} K(x, y ; \xi, \eta) d \xi d \eta=1$. We then focus our attention on integrals of the following form

$$
T(\omega)=\iiint \int_{-\infty}^{+\infty} K(x, y ; \xi, \eta)[\omega(x, y)-\omega(\xi, \eta)]^{2} d x d y d \xi d \eta
$$

taken over the entire plane, and investigate the problem $P_{n}$,

$$
T(\omega)=\min .,
$$

for which the admissible functions are prescribed in the domain $\bar{B}$, complementary to $B$, that is, in the portion of the plane outside of $B$.

For example, if $K=f(r)$, where $r^{2}=(x-\xi)^{2}+(y-\eta)^{2}$ and $f(r) \geqq 0$, while $f(r)=0$ for $r>h$, it is easily verified that, as the parameter $h$ tends to zero, we have $T(\omega) \rightarrow D(\omega)$ so that $P_{n}$ tends to the boundary value problem for $\Delta u=0$. It can be shown generally that the nucleus $K$ may be chosen dependent on a parameter $h$ so that $T(\omega)$ tends to a given quadratic functional which yields as Euler equation any prescribed homogeneous elliptic differential equation.

Now the variational condition for the problem (16a) is no longer a differential equation but a Fredholm integral equation, which is more easily treated:

$$
u(x, y)=\iint_{B} K(x, y ; \xi, \eta) u(\xi, \eta) d \xi d \eta+g(x, y),
$$

where

$$
g(x, y)=\iint_{\bar{B}} K(x, y ; \xi, \eta) u(\xi, \eta) d \xi d \eta
$$


is known because $u$ is prescribed in $\bar{B}$. The solution $u_{n}$ of (17) for $K=K_{n}$ converges to the solution $u$ of the problem $P$. Moreover, and this is the important point for practical applications, it can be proved that the integral equation (17) is solvable by the Neumann method of iteration under very wide conditions for the nucleus $K$. Thus we may develop a new method for attacking boundary value problems which, however, so far has not been tested in practical applications.

It may be remarked that, generally speaking, the iteration process will converge more slowly if the approximation of $P_{n}$ to $P$ becomes better. Hence again a compromise for practical purposes is indicated.

In passing, we mention the intimate connection of this method with statistics. Let us suppose a substance to be distributed with a density $u(x, y)$ over the plane. We now imagine that the substance is redistributed in distinct steps in such a manner that a unit mass concentrated at the point $P(x, y)$ will be spread over the plane with a density $K(x, y ; \xi, \eta)$ at the point $Q(\xi, \eta)$. The integral equation (17) then characterizes a state of statistical equilibrium, if the density $u(x, y)$ is prescribed in the complementary domain $\bar{B}$ of $B$. Of course, if we interpret the integrals as Stieltjes' integrals, we may include in our formulation even problems of finite differences and random walk problems of the classical type. For us here, the main objective is to point out a method that enables us in principle to find approximately solutions of boundary value problems.

\section{Method OF GRADIENTS}

Still another alternative to the Rayleigh-Ritz procedure should be mentioned. This is the method of gradients, which goes back to a paper published by Hadamard in 1907 [12]. Highly suggestive as is Hadamard's attempt, difficulties of convergence were encountered. However, recent developments in the theory of conformal mapping and in Plateau's problem throw new light on Hadamard's idea, so that it seems justified now to expect from it not only purely mathematical existence proofs but also a basis for numerical treatment in suitable cases $[13,10,11]$.

The principle of the method may be understood from the elementary geometric concept of a vector gradient. Let $u=f\left(x_{1}, \cdots, x_{n}\right)$ be a non-negative function of the $n$ variables $x_{i}$, or as we might say of the position vector $X=\left(x_{1}, \cdots, x_{n}\right)$, and let us seek to determine a vector $X=X_{0}$ for which $u$ is at least stationary. We then proceed as follows: on the surface $u=f(x)$ we move a point $\left(x_{1}, \cdots, x_{n}, u\right)$ so that $x_{i}(t)$ and $u(t)$ become functions of a time-parameter $t$. Then 
the velocity of ascent or descent along the line $X=X(t), u=u(t)$ on the surface is

$$
\frac{d u}{d t}=\dot{u}=\sum_{i=1}^{n} \dot{x}_{i} f_{x_{i}}=\dot{X} \cdot \operatorname{grad} f .
$$

We now choose the line along the line along which the motion proceeds so that the descent is as steep as possible (lines of steepest descent). This means to make $u$ negative and as large "as possible" in absolute value, for example, by choosing

$$
\dot{X}=-\operatorname{grad} f
$$

so that

$$
\dot{u}=-(\operatorname{grad} f)^{2} .
$$

Hence the position vector $X$ moves according to the system of ordinary differential equations (18) along the lines of steepest descent with respect to the function $f$. Under very general assumptions, it is clear that $X$, starting from an arbitrary initial position, will, for $t \rightarrow \infty$, approach a position for which $\operatorname{grad} f=0$, and therefore for which $f$ is stationary and possibly a minimum. However, instead of using the continuous procedure given by the differential equation (18), we may proceed stepwise, correcting a set of approximations $x$ to the solutions of the equations grad $f=0$ by corrections proportional to the respective components of $-\operatorname{grad} f$.

This elementary idea can be generalized to variational problems. If we wish to determine a function $u(x, y)$ defined in $B$ and having prescribed boundary values such that $u$ is the solution of a variational problem

$$
I(v)=\iint_{B} F\left(x, y, v, v_{x}, v_{y}\right) d x d y=\min .,
$$

then we interpret the desired function $u$ as the limit for $t \rightarrow \infty$ of a function $v(x, y, t)$, whose values may be chosen arbitrarily for $t=0$ and for all $t$ thereafter are determined in such a way that the expression $I(v)$, considered as a function $I(t)$ of $t$, decreases as rapidly as possible toward its minimal value. Of course the boundary values of $v(x, y, t)$ are the same as those for $u(x, y)$, so that $v_{t}$ must vanish at the boundary. If we choose $v=v(x, y, t)$, we find

$$
\dot{I}(t)=-\iint_{B} v_{t} L(v) d x d y,
$$


where $L(v)$ is the Euler expression corresponding to (20). To consider a concrete example, we suppose that

$$
I(v)=\iint_{B}\left(v_{x}^{2}+v_{y}^{2}\right) d x d y,
$$

so that our minimum problem amounts to determining the equilibrium of a membrane with given boundary deflections $g(s)$. Then $L(v)=-2 \Delta v$. Incidentally (20) displays an analogy between the Euler expression and the gradient of a function $f\left(x_{1}, \cdots, x_{n}\right)$ of $n$ independent variables. The variation or "velocity" of $I(v)$ is expressed as an "inner product" of the velocity of the "independent function" $v$ with the Euler expression $L(v)$, the gradient of a functional in function space.

We now assure ourselves of a steady descent or decrease of $I(t)$ by choosing $v_{t}$ in accordance with the differential equation

$$
v_{t}=-k L(v)
$$

where $k$ is a positive arbitrary function of $x, y$. (21) then becomes

$$
I(t)=-\iint_{B} k[L(v)]^{2} d x d y,
$$

and again we can infer that, for $t \rightarrow \infty, v(x, y, t)$ will tend to the solution $u(x, y)$ of the corresponding boundary value problem $L(u)=0$.

For the case of the membrane the differential equation (22) becomes

$$
v_{t}=\Delta v,
$$

the equation of heat transfer. In our interpretation this equation describes a rapid approach to a stationary state along the "lines of steepest descent." While for the equations (23) or (22) the convergence of $v$ for $t \rightarrow \infty$ can be proved, serious difficulty arises if we want to replace our continuous process by a process of stepwise corrections as would be required for numerical applications. Each step means a correction proportional to $\Delta v$, thus introducing higher and higher derivatives of the initial function $v$. Another great difficulty is presented by rigid boundary values. ${ }^{7}$

Yet there do exist classes of problems where such difficulties can be overcome if the method is extended properly. First of all we may

7 Incidentally, if we apply this procedure to a problem for a finite net, it converges very well and is, as a matter of fact, nothing but a natural method of solving a system of linear equations by a method of iteration. 
observe that it is not necessary to select the steepest descent along the gradient; it suffices to secure a safe descent at a suitably fast rate. Furthermore, if we consider problems for which the boundary value problem of the differential equation presents no difficulties for the domain $B$, but for which a degree of freedom in the boundary values is left, then the problem reduces to one for finding these boundary values, and now all our difficulties disappear. A typical example is the problem of the conformal mapping of a circle $B$ onto a simplyconnected domain $G$, and it may be that the method of gradients opens a path for the attack of the problem of conformal mapping for multiply-connected domains and other problems as well.

This address has emphasized more theoretical aspects. However, some of the general principles described may be helpful in enlarging our equipment for practical purposes.

\section{ApPENDIX $^{8}$}

\section{NUMERICAL TREATMENT OF THE PLANE TORSION PROBLEM FOR MULTIPLY-CONNECTED DOMAINS}

The computation of the stiffness $S$ defined in $\$ I, 2$ a furnishes an example of independent interest which permits to compare the practical merits of some of the methods described in this address. Numerical calculations were carried out for the cross sections of the following diagrams, a square from which a smaller square is cut out; and a square, from which four squares are cut out. In the first case our quadratic frame was supposed to be bounded by the lines $x= \pm 1$, $y= \pm 1$ and $x= \pm 3 / 4, y= \pm 3 / 4$. To apply the Rayleigh-Ritz method for the domain as a whole would already be cumbersome because of the boundary conditions for admissible functions $\phi$. However, this difficulty disappears if we exploit the symmetry of the domain and the resulting symmetry of the solution; thus we may confine ourselves to considering only one-eighth of the domain $B^{*}$, namely the quadrangle $A B C D$. For this polygon any function of the type

$$
\phi=a(1-x)[1+(x-3 / 4) P]
$$

where $P(x, y)$ is a polynomial, is admissible, and its substitution in the integral leads to simple linear equations for the cofficients. Thus for the simplest attempt

$$
\phi=a(1-x)
$$

which leaves only one constant $a$ to be determined, we find with a

${ }^{8}$ Addition not contained in the original address. 
negligible amount of numerical labor $S=.339$ and $c=-.11$. A refined attempt with the function

$$
\phi=a(1-x)[1+\alpha(x-3 / 4) y]
$$

yielded $S=.340$ and $c=-.109$ with little more labor.

These results were checked with those obtained by our generalized method of finite differences where arbitrary triangular nets are permitted. The diagrams are self-explanatory. Unknown are the

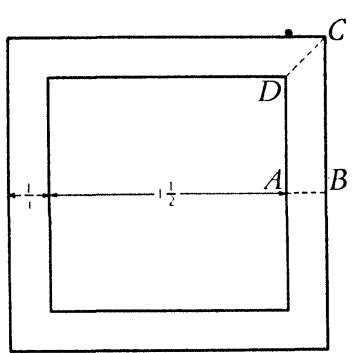

FIG. 2

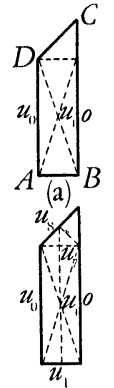

(c)

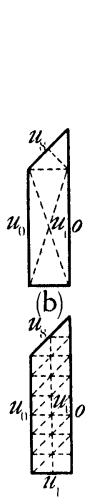

(d)

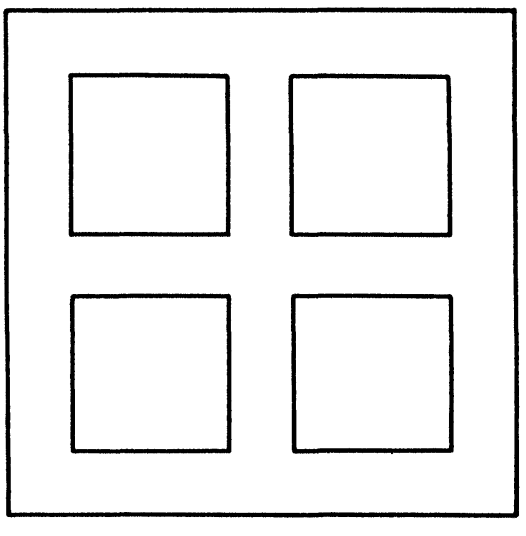

FIG. 3

net-point-values $u_{i},\left(c=u_{0}\right)$. In the net-triangles our functions were chosen as linear, so that the variational problem results in linear equations for the $u_{i}$. The results, easily obtainable, were: case (a) with two unknowns: $S=.344, u_{0}=-.11$; case (b) with three unknowns: $S=.352, u_{0}=-.11$; case (c) with five unknowns $S=.353$, $u_{0}=-.11$; case $(\mathrm{d})$ with nine unknowns, corresponding to the ordinary difference method $S=.353, u_{0}=-.11$.

These results show in themselves and by comparison that the generalized method of triangular nets seems to have advantages. It was applied with similar success to the case of a square with four holes, and it is obviously adaptable to any type of domain, much more so than the Rayleigh-Ritz procedure in which the construction of admissible functions would usually offer decisive obstacles.

In a separate publication it will be shown how the method can be extended also to problems of plates and to other problems involving higher derivatives.

Of course, one must not expect good local results from a method 
using so few elements. However, it might be expected that a smooth interpolation of the net functions obtained will yield functions which themselves with their derivatives are fairly good approximations to the actual quantities.

\section{REFERENCES}

1. R. Courant, Ueber direkte Methoden bei Variations- und Randwertproblemen, Jber. Deutschen Math. Verein. vol. 34 (1925) p. 90 ff.; reprinted in Math. Ann. vol. 97 (1927) p. $711 \mathrm{ff}$.

2. - Ueber direkte Methoden ..., Proceedings of the International Congress for Applied Mechanics, Delft, 1925.

3. - Ueber partielle Differenzengleichungen, Atti del Congresso Internazionale dei Matematici, Bologna, 1928.

4. - Ueber Randwertaufgaben bei partiellen Differenzengleichungen, Zeitschrift für angewandte Mathematik und Mechanik vol. 6 (1926) p. $1 \mathrm{ff}$.

5. - Ueber die Theorie der linearen partiellen Differenzengleichungen, Goettingen Nachrichten, 1925.

6. - Bemerkungen zur numerischen Aufloesung von Randwertproblemen ..., Goettingen Nachrichten, 1926, p. $111 \mathrm{ff}$.

7. - Ueber ein convergenzerzeugendes Prinzip der Variationsrechnung, Goettingen Nachrichten, 1922.

8. R. Courant, K. Friedrichs, H. Lewy, Ueber die partiellen Differenzengleichungen der mathematischen Physik, Math. Ann. vol. 100 (1928) p. $32 \mathrm{ff}$.

9. R. Courant and D. Hilbert, Methoden der mathematischen Physik, vol. I, 2d edition, 1931; vol. II, 1937.

10. R. Courant, On a method for the solution of boundary-value problems, Theodore von Kármán Anniversary Volume, 1941.

11. - On the first variation of the Dirchlet-Douglas integral and on the method of gradients, Proc. Nat. Acad. Sci. U.S.A. vol. 27 (1941) p. $242 \mathrm{ff}$.

12. J. Hadamard, Mémoire sur le problème d'analyse relatif a l'équilibre des plaques élastiques encastrées, Mémoires presentés par divers savants éstrangers à l'Académie des Sciences de l'Institut de France (2) vol. 33, 4 (1908).

13. H. Lewy, Ueber einen Ansatz zur numerischen Loesung von Randwertproblemen, Goettingen Nachrichten, 1925, p. $118 \mathrm{ff}$.

14. Philipps and N. Wiener, Nets and the Dirichlet problem, Publication of the Massachusetts Institute of Technology, 1925.

15. R. G. D. Richardson, $A$ new method in boundary value problems for differential equations, Trans. Amer. Math. Soc. vol. 18 (1917) p. 489 ff.

16. R. Lueneburg, Das Problem der Irrfahrt ohne Richtungsbeschraenkung . . , Math. An n. vol. 104 (1931) p. $700 \mathrm{ff}$.

17. Petrovsky, Ueber das Irrfahrtenproblem, Math. Ann. vol. 109 (1934) p. $425 \mathrm{ff}$.

18. W. Feller, Zur Theorie der Stochastischen Prozesse, Math. Ann. vol. 113 (1936) p. $113 \mathrm{ff}$.

19. C. J. Thorne and J. V. Atanasoff, A functional method for the solution of thin plate problems . . , Iowa State College Journal of Science vol. 14 (1940) p. $333 \mathrm{ff}$.

20. A. Weinstein, Etude des spectres des équations aux dérivées partielles de la théorié des plaques élastiques, Mémorial des Sciences Mathématiques, no. 88, 1937.

21. - On a minimal problem in the theory of elasticity, J. London, Math. Soc. vol. 10 (1935) p. $184 \mathrm{ff}$. 
22. - Les vibrations et le calcul des variations, Portugaliae Mathematica, 1941, p. 36.

23. E. Trefftz, Ueber Fehlerabschätzungen bei Berechnung von Eigenwerten, Math. Ann. vol. 108 (1933) p. 595 ff.

24. - Konvergenz und Fehlerabschaetzung beim Ritz'schen Verfahren, Math. Ann. vol. 100 (1928) p. 503 ff.

25. - Ein Gegenstueck zum Ritz'schen Verfahren, Verhandlungen, Congress für technische Mechanik, Zuerich, 1927, p. 131.

26. $\mathrm{H}$. Cross, Analysis of continuous frames by distributing fixed-end moments, Transactions of the American Society of Civil Engineers vol. 96 (1932) pp. 1-10.

27. R. V. Southwell, Relaxation methods in engineering science. A treatise on approximate computations, Oxford, 1940.

28. K. Friedrichs, Ein Verfahren der Variationsrechnung . . , Goettingen Nachrichten, 1929, p. $13 \mathrm{ff}$.

29. K. Friedrichs and G. Horvay, The finite Stieltjes momentum problem, Proc. Nat. Acad. Sci. U.S.A. vol. 25 (1939) pp. 528-534.

30. L. Collatz, Das Differenzenverfahren . . . , Schriften des Mathematisches Seminars der Universitaet Berlin, vol. 3 (1935).

31. - Ueber das Differenzenverfahren..., Zeitschrift für angewandte Mathematik und Mechanik vol. 16 (1936) p. $239 \mathrm{ff}$.

32. - Bemerkungen zur Fehlerabschaetzung für das Differenzenverfahren bei partiellen Differentialgleichungen, Zeitschrift für angewandte Mathematik und Mechanik vol. 13 (1933) p. $56 \mathrm{ff}$.

33. H. P. Geiringer, Zur Praxis der Loesung linearer Gleichungen in der Statik, Zeitschrift für angewandte Mathematik und Mechanik vol. 8 (1928) p. $446 \mathrm{ff}$.

34. R. von Mises and H. P. Geiringer, Praktische Verfahren der Gleichungsaufloesung, Zeitschrift für angewandte Mathematik und Mechanik vol. 9 (1929) pp. 58-77; 152-164.

35. J. P. Den Hartog, Mechanical vibrations, 1st edition, 1934, New York.

36. S. Gerschgorin, Fehlerabschaetzung fuer das Differenzenverfahren zur Loesung partieller Differentialgleichungen, Zeitschrift für angewandte Mathematik und Mechanik vol. 10 (1930) pp. 373-382.

37. S. Timoshenko, Vibration problems in engineering, 2d edition, New York, 1938.

38. Lord Rayleigh, Theory of sound, 2d edition, 1894 and 1896, London.

39. W. Ritz, Ueber eine neue Methode zur Loesung gewisser Variationsprobleme der mathematischen Physik, J. Reine Angew. Math. vol. 135 (1908); Theorie der Transversalschwingungen einer quadratischen Platte mit freien Raendern, Annalen der Physik vol. 38 (1909).

40. Si Luan Wei, Ueber die eingespannte rechteckige Platte . . , dissertation, Goettingen, 1925.

\section{NeW York UNIVERSITY}

